\title{
The Capacity Constrained Facility Location problem*
}

\author{
HARIS AZIZ, UNSW Sydney and Data61 CSIRO \\ HAU CHAN, University of Nebraska-Lincoln \\ BARTON E. LEE, UNSW Sydney and Data61 CSIRO \\ DAVID C. PARKES, Harvard University
}

\begin{abstract}
We initiate the study of the capacity constrained facility location problem from a mechanism design perspective. The capacity constrained setting leads to a new strategic environment where a facility serves a subset of the population, which is endogenously determined by the ex-post Nash equilibrium of an induced subgame and is not directly controlled by the mechanism designer. Our focus is on mechanisms that are ex-post dominant-strategy incentive compatible (DIC) at the reporting stage. We provide a complete characterization of DIC mechanisms via the family of Generalized Median Mechanisms (GMMs). In general, the social welfare optimal mechanism is not DIC. Adopting the worst-case approximation measure, we attain tight lower bounds on the approximation ratio of any DIC mechanism. The well-known median mechanism is shown to be optimal among the family of DIC mechanisms for certain capacity ranges. Surprisingly, the framework we introduce provides a new characterization for the family of GMMs, and is responsive to gaps in the current social choice literature highlighted by Border and Jordan (1983) and Barbarà, Massó and Serizawa (1998).
\end{abstract}

\section{INTRODUCTION}

A common economic problem is deciding where a public facility should be located to service a population of agents with heterogenous preferences. For example, a government needs to decide the location of a public hospital, or library. More abstractly, the 'location' may represent a type or quality of a service. For example, a government may have a fixed hospital location but must decide on the type of service the hospital will specialize, and, in particular, whether the service will be targeted to those suffering from acute, moderate, or mild severity of a certain illness. In such problems, participants may benefit by misreporting their preferences, and this can be problematic for a decision maker trying to find a socially optimal solution. This leads to the mechanism design problem of providing optimal, or approximately optimal, solutions while also being strategyproof, i.e., no agent can profit from misreporting their preferences regardless of what others report. ${ }^{1} \mathrm{We}$ call this the facility location problem.

A large literature has studied the facility location problem under the assumption that the facility does not face capacity constraints. When the facility is not capacity constrained, all agents can benefit from the facility and hence it is modeled as a public good. ${ }^{2}$ Under this assumption, the mechanism design problem is explored in several classic papers [Black, 1948, Border and Jordan, 1983, Gibbard, 1973, 1977, Moulin, 1980, Satterthwaite, 1975], and more recently in algorithmic mechanism design [Feldman et al., 2016, Nisan and Ronen, 2001, Procaccia and Tennenholtz, 2013].

\footnotetext{
*This paper was previously circulated under the title "Mechanism Design without Money for Common Goods." Authors' email addresses: Haris Aziz: haris.aziz@unsw.edu.au; Hau Chan: hchan3@unl.edu, Barton E. Lee: barton.e.lee@gmail.com, David C. Parkes: parkes@eecs.harvard.edu.

${ }^{1}$ We focus on the 'mechanism design without money' problem where the use of money is assumed to not be permitted. This is a natural assumption for environments where the use of money is considered unlawful (e.g., organ donations) or unethical (e.g., political decision making, or locating a public good).

${ }^{2} \mathrm{~A}$ public good is non-rivalrous and non-excludable.
} 
To the best of our knowledge, an unexplored setting for the mechanism design problem is where the public facility is capacity constrained. ${ }^{3}$ Capacity constraints limit the number of agents who can benefit from the facility's services. Such constraints are ubiquitous in practice: a hospital is capacity constrained by the number of beds and doctors, and a library may have limited seating. When present, capacity constraints introduce a particular form of rivalry to the facility, since once the facility reaches its capacity limit additional agents are prevented from using, and hence benefiting, from the facility.

A number of new strategic challenges arise for the mechanism designer when the public facility is capacity constrained but is still non-excludable. For example, when the mechanism designer chooses a location for the facility, we cannot stipulate which agents will be served, instead these decisions are made by participants, through strategic interactions once the facility has been located. That is, the ex-post Nash equilibrium of a subgame induced by the facility location determines the agents who ultimately benefit from the facility and those who do not. This introduces a technical challenge, because it leads agents to have interdependent utilities, since the utility for a particular location depends on who else will use the location (and in turn on their preferences). Furthermore, the reporting game is made in anticipation of the extensive-form game and ex-post Nash equilibrium, and the designer must consider mechanisms that are strategyproof in this broader game-theoretic context.

In this paper, we initiate the study of the capacity constrained facility location problem from the viewpoint of mechanism design. In our model, $n$ agents are located in the $[0,1]$ interval, and there is a single facility to be located, this facility is able to service at most $k$ agents, where $k$ is some positive integer. When $k \geq n$ the capacity constraint is of no effect, and the capacity constrained facility location problem is equivalent to the classic problem. Agent locations are privately known, and, given a facility location, the ex-post Nash equilibrium of an induced subgame determines which agents are served. The mechanism designer's problem is to design mechanisms that are strategyproof and maximize social welfare. In our model, we take strategyproof to mean ex-post dominant-strategy incentive compatible (DIC) at the reporting stage. That is, conditional on the ex-post Nash equilibrium being attained in the induced subgame, an agent never benefits ex-post from misreporting their location to the mechanism regardless of what other agents report, and regardless of other agents' true locations. For ease of exposition, a mechanism that is DIC at the reporting stage will simply be said to be DIC. Unlike the classic facility location problem where the facility is not capacity constrained, the social welfare optimal mechanism is not DIC except when the capacity constraint is trivial, i.e., $k=1$ or $n$. As a result, we follow the approach of Procaccia and Tennenholtz [Procaccia and Tennenholtz, 2013] and consider the approximate mechanism design problem. We adopt the worst-case approximation measure for social welfare, and ask what is the best approximation achievable with a DIC mechanisms and how does this vary as a function of the capacity constraint?

The literature studying the facility location problem without capacity constraints, or simply $k=$ $n$, provides a number of important results. Gibbard-Satterthwaite [Gibbard, 1973, Satterthwaite, 1975] showed a powerful impossibility result: when agents can have unrestricted preferences there need not exist any strategyproof mechanism. As a result, more recent works typically restrict agent preferences' over the location of the facility to be single-peaked and sometimes in addition

\footnotetext{
${ }^{3}$ There is a distinct setting sometimes referred to as the 'constrained facility location' problems [Sui and Boutilier, 2015] where the feasible locations for the facility are constrained. The algorithmic problem, of locating multiple capacity constrained facilities when agents are not strategic, has also been studied [Brandeau and Chiu, 1989, Pál et al., 2001, Vygen, 2004].
} 
symmetric. ${ }^{4}$ We focus on the case where, conditional on the agent being served, the agent has preferences that are both single-peaked and symmetric. When the objective of the mechanism designer is to maximize social welfare, i.e., utilitarian welfare, the standard median mechanism is both strategyproof and social welfare optimal [Black, 1948]. More generally, a goal of the social choice literature has been to characterize the complete family of strategyproof mechanisms. Closest to our setting, Border and Jordan [Border and Jordan, 1983] provide a partial characterization of strategyproof mechanisms via the family of Generalized Median Mechanisms (GMMs). Border and Jordan show that a mechanism is strategyproof and unanimity respecting ${ }^{5}$ if and only if it is a GMM, and that the family of GMMs is strictly smaller than the complete family of strategyproof mechanisms. ${ }^{6}$ This has left a gap in the literature to characterize the complete family of strategyproof mechanisms and understand the difference in strategyproof mechanisms that are GMMs and those that are not. Figure 2 schematically illustrates this gap.

Our Contributions: We introduce a new mechanism problem, the capacity constrained facility location problem. This problem is a natural variant of the classic facility problem where the facility is assumed to face capacity constraints. A conceptual contribution is to formalize the effect of capacity constraints when the facility is non-excludable but cannot service all agents. We do this by defining an extensive-form game involving the mechanism designer and agents. First, agents report their preferences to the designer, and then the facility is located by the mechanism. Once the facility is located a subgame is induced where agents strategically choose whether or not to attempt to be served by the facility. The ex-post Nash equilibrium determines which agents are served by the facility and which are not. We seek mechanisms that are strategyproof in this broader gametheoretic context, i.e., ex-post dominant-strategy incentive compatible; that is, conditional on the ex-post Nash equilibrium being achieved in the subgame, no agent can benefit from misreporting their location regardless of what other agents report and regardless of other agents' true locations.

Our main theoretical contribution is a complete characterization of DIC mechanisms for the capacity constrained facility location problem. We show that a mechanism is DIC if and only if it belongs to the established family of mechanisms called the Generalized Median Mechanisms (GMMs), which appear in Moulin [Moulin, 1980] and Border and Jordan [Border and Jordan, 1983] Thus, the framework we introduce surprisingly provides a new characterization of GMMs. This result contributes to a novel perspective to a "major open question" (Barbarà, Massó and Serizawa [Barbarà et al., 1998]) posed in Border and Jordan [Border and Jordan, 1983] (further discussion is provided in Section 2).

We also provide algorithmic results and study the performance of DIC mechanisms in optimizing social welfare. We adopt the worst-case approximation measure, and provide a lower bound on the approximation ratio of any DIC mechanism. We show that at best the approximation ratio of a DIC mechanism is $2 \frac{k}{k+1}$ when $k \leq\lceil(n-1) / 2\rceil$, and $\max \left\{\frac{n-1}{k+1}, 1\right\}$ otherwise. Interestingly, this lower bound is achieved by the standard median mechanism (which is also DIC) when $k \leq\lceil(n-1) / 2\rceil$ or $k=n$, and hence the median mechanism is optimal among all DIC mechanisms in those ranges. Figure 1 illustrates these approximation results.

\footnotetext{
${ }^{4}$ A single-peaked preference is symmetric if equidistant locations on either side of the ideal, or 'peak', location are always equally preferred.

${ }^{5}$ Unanimity respecting simply means that if there is a unanimously most preferred facility location then the mechanism must locate the facility at this location.

${ }^{6}$ We note that in a slightly different setting, where the single-peaked preferences are possibly asymmetric, GMMs provide a complete characterization of strategyproof and 'peak only' mechanisms (Proposition 3 of Moulin [Moulin, 1980]). Example 3.3 in the present paper provides an example of a mechanism that is strategyproof in the Border and Jordan [Border and Jordan, 1983] setting but not the Moulin [Moulin, 1980] setting.
} 
Finally, we consider an extension of our framework where the mechanism designer can also restrict access to the facility, and hence dictate which agents are served. This extension is relevant to settings where the designer can issue permits, and prevent certain agents from accessing the facility. Under an anonymity assumption, we show that no mechanism that both locates the facility and stipulates which agents can be served is DIC.

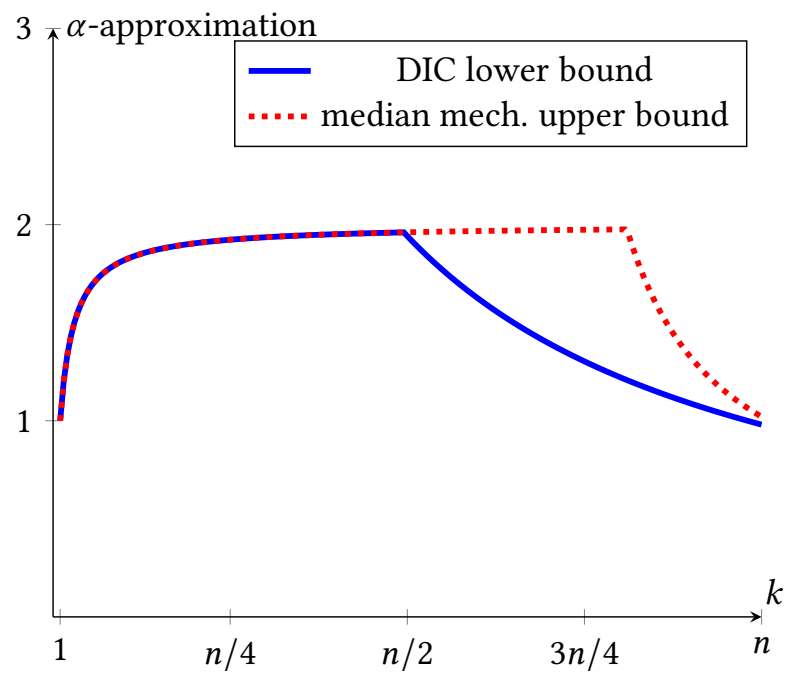

Fig. 1. Worst-case approximation ratio as a function of the capacity constraint, $k$.

Outline: Section 2 provides a brief literature review. Section 3 presents our model and formalizes the objective of the mechanism designer, Section 3.1 then presents our key characterization result of DIC mechanisms. Section 4 explores the performance, i.e., approximation results, of DIC mechanisms. Section 5 considers an extension of our framework where the mechanism designer is able to dictate which agents are served by the facility. Lastly, we conclude with a discussion in Section 6.

\section{RELATED LITERATURE}

A number of papers have considered related mechanism problems where the use of money is not permitted [Abdulkadiroğlu and Sönmez, 2003, Ashlagi and Roth, 2011, Border and Jordan, 1983, Gibbard, 1973, Mei et al., 2016, Moulin, 1980, Procaccia and Tennenholtz, 2013, Satterthwaite, 1975, Sui and Boutilier, 2015]. Most closely related to our paper is [Procaccia and Tennenholtz, 2013], where agents with single-peaked preferences are located along the real line and the problem of locating a (non-capacity constrained) public facility is studied with the goal of minimizing two distinct objective functions; the total social cost and the maximum social cost. This problem is often referred to as a single facility location problem, or single facility location game. ${ }^{7}$ In this paper, we focus on minimizing the first objective function in the new environment where the facility

\footnotetext{
${ }^{7}$ We do not review a large segment of computer science and operations research literature on facility location problems that assumes complete information and hence does not require a mechanism design approach to overcome strategic tensions (for a survey see [Brandeau and Chiu, 1989]). Furthermore, this literature, when incorporating capacity constraints, typically focuses on the problem of locating multiple capacity constrained facilities that have sufficient capacity to service all agents [Charikar et al., 2002, Cygan et al., 2012, Pál et al., 2001, Vygen, 2004]. Instead we review the subset of literature that assumes strategic agents and takes a mechanism design approach.
} 
is capacity constrained. In contrast to the setting studied by [Procaccia and Tennenholtz, 2013], agents have interdependent utilities, in our model, due to the capacity constraints of the facility and the induced subgame. Accordingly, the mechanism design problem requires consideration of a broader game-theoretic environment where agents face an extensive-form game when reporting preferences.

Another large body of literature has been concerned with characterizing DIC mechanisms for the unconstrained facility location problem. The key pioneering works in this area are by Moulin [Moulin, 1980], and Border and Jordan [Border and Jordan, 1983]. In one-dimensional space and for symmetric and single-peaked preferences, Border and Jordan [Border and Jordan, 1983] characterize a general class of DIC mechanisms which have become to be known as generalized median mechanisms (GMM), and in addition show that when the property of unanimity is enforced every DIC mechanism is a GMM. ${ }^{8}$ These results differ slightly from the characterization results of Moulin [Moulin, 1980] since the setting studied in [Moulin, 1980] does not restrict the single-peaked preferences to be symmetric. Characterizing DIC but non-unanimity respecting mechanisms was posed as an open problem; as stated by Border and Jordan in [Border and Jordan, 1983] "[the characterization] leaves several open problems. The most obvious question is: what happens if the unanimity assumption is dropped?" Characterizations however, have remained elusive and it has become known as a "major open question" [Barbarà et al., 1998] with only partial progress towards a resolution [Barbarà et al., 1998, Ching, 1997, Peremans et al., 1997, Weymark, 2011]. In this paper we focus on the one-dimensional case where open questions still remain; in particular, the results of [Border and Jordan, 1983] in one-dimensional space leaves two gaps:

(1) there exist non-unanimity respecting DIC mechanisms that are not GMM, and

(2) there exist DIC mechanisms that are GMMs but do not respect unanimity.

Our characterization of DIC mechanisms via the family of GMM, although considered in a different setting where the facility is capacity constrained, applies more generally to mechanisms that are not unanimity respecting. Hence, we contribute a novel perspective to these gaps in characterization, showing that a mechanism is DIC for all possible capacity constraint $k \leq n$ if and only if it is a GMM. This means that any mechanism in gap (1) is not DIC when the facility is capacity constrained with $k<n$. Furthermore, the unanimity property is sufficient to ensure that a mechanism that is DIC in the non-capacity constrained setting remains DIC when capacity constraints are present.

\section{MODEL, BASIC PROPERTIES, AND DEFINITIONS}

Model: Let $N=\{1, \ldots, n\}$ be a finite set of $n$ agents and let $X=[0,1]$ be the domain of agent locations. Each agent $i \in N$ has a location $x_{i} \in X$, which is privately known, the profile of agent locations is denoted by $\boldsymbol{x}=\left(x_{1}, x_{2}, \ldots, x_{n}\right)$. The profile of all agent except some agent $i \in N$ is denoted by $\boldsymbol{x}_{-i}=\left(x_{1}, x_{2}, \ldots, x_{i-1}, x_{i+1}, \ldots, x_{n}\right)$. There is a single facility to be located in $X$ by some mechanism. A mechanism is a function $M: \prod_{i \in N} X \rightarrow X$ mapping a profile of locations to a single location. ${ }^{9}$ We denote the mechanism's output, or facility location, by $s \in X$.

The facility faces a capacity constraint $k: k \leq n$, which provides a limit on the number of agents that can be served. A served agent attains utility $u_{i}=1-d\left(s, x_{i}\right) \geq 0$, where $d(\cdot, \cdot)$ denotes the Euclidean metric; an unserved agent attains zero utility, $u_{i}=0 .{ }^{10}$ The set of agents served

\footnotetext{
${ }^{8}$ Border and Jordan [Border and Jordan, 1983] also consider the problem in higher dimensions.

${ }^{9}$ We restrict our attention to deterministic mechanisms.

${ }^{10}$ Our characterization results (Section 3.1) do not rely on this specific utility function - we only require that agents weakly prefer to be served than not, and conditional on being served the agent's utility is symmetric and (strictly) single-peaked. However, our approximation results do rely on the choice of utility function.
} 
by the facility's limited capacity is not directly controlled by the mechanism, since the facility is assumed to be non-excludable. ${ }^{11}$ Instead, this is determined by the equilibrium outcome of a subgame induced by the mechanism's choice of facility location.

Given an instance $\langle\boldsymbol{x}, s, k\rangle$, we assume that the set of agents served by the facility is determined via the ex-post Nash equilibrium ${ }^{12}$ of a subgame, $\Gamma_{x}(s, k)$. The subgame $\Gamma_{x}(s, k)$ is as follows. Each agent $i \in N$ chooses an action $a_{i} \in A=\{\emptyset, s\}$ of whether, or not, to travel from their location $x_{i}$ to the facility location $s$. Action $a_{i}=s$ denotes agent $i$ 's choice to travel to the facility, and action $a_{i}=\emptyset$ denotes the agent's choice to not travel to the facility. We denote the profile of agent actions by $\boldsymbol{a}=\left(a_{1}, a_{2}, \ldots, a_{n}\right)$. An agent $i$ is served by the facility if they travel to the facility, $a_{i}=s$, and strictly less than $k$ other agents travel to the facility, i.e., $|N(\boldsymbol{a}, s)| \leq k$ where $N(\boldsymbol{a}, s):=\left\{i \in N: a_{i}=s\right\}$. If instead they travel to the facility and at least $k$ other agents also travel to the facility, i.e., $|N(\boldsymbol{a}, s)|>k$, then a tie-breaking rule is used to determine which subset of $k$ agents in $N(\boldsymbol{a}, s)$ are served. We assume a distance-based tie-breaking rule ( $\triangleright)$ whereby agent $i$ has higher priority than agent $j$, denoted $i \triangleright j$, if agent $i$ is closer to the facility than agent $j$, i.e., $d\left(s, x_{i}\right)<d\left(s, x_{j}\right)$; if agent $i$ and $j$ are equidistant, i.e., $d\left(s, x_{i}\right)=d\left(s, x_{j}\right)$, then we apply some deterministic tie-breaking rule. ${ }^{13}$ This distance-based tie-breaking rule can be motivated by a 'first-come-first-serve' protocol when the location $s$ is geographical and agents physically travel to the facility to be served. If the location $s$ corresponds to a type, or quality, of service the 'firstcome-first-serve' protocol is analogous to a 'best-fit' tie-breaking protocol that prioritizes agents according to how close the type of service being offered is to their true needs, i.e., $d\left(s, x_{i}\right)$. An agent $i$ with location $x_{i}$ attains utility $1-d\left(s, x_{i}\right)$ if $a_{i}=s$ and they are served, if $a_{i}=s$ and they are not served they attain utility $-d\left(s, x_{i}\right)$, and otherwise $a_{i}=\emptyset$ and agent $i$ attains zero utility.

Abusing terminology slightly, given a profile of locations $\boldsymbol{x}$ and facility location $s$, we will refer to $k$ highest priority agents with respect to the distance-based tie-breaking rule $(\triangleright)$ as the $k$-closest agents. We denote this set of agents by $N_{k}^{*}(\boldsymbol{x}, s)$.

Basic properties of the model: For any instance $\langle\boldsymbol{x}, s, k\rangle$, the subgame $\Gamma_{\boldsymbol{x}}(s, k)$ has an (essentially) unique equilibrium. There always exists an equilibrium where the $k$-closest agents, $N_{k}^{*}(\boldsymbol{x}, s)$, choose to travel to the facility and are served by the facility. In instances where one or more of the $k$-closest agents are indifferent between being served and not traveling to the facility, i.e., whenever $d\left(s, x_{i}\right)=1$ for some $i \in N_{k}^{*}(\boldsymbol{x}, s)$, multiple equilibria arise. For the purposes of this paper these equilibria are all 'equivalent' since every agent attains the same utility in each of the equilibria. Proposition 3.1 states this basic property. The proof is straightforward and left to the appendix for the interested reader.

Proposition 3.1. For any instance $\langle\boldsymbol{x}, s, k\rangle$, there exists an equilibrium of the subgame $\Gamma_{\boldsymbol{x}}(s, k)$ and, furthermore, in every equilibrium agent $i \in N$ attains utility $1-d\left(s, x_{i}\right)$ if $i \in N_{k}^{*}(x, s)$, and otherwise, attains zero utility.

Given Proposition 3.1, we can denote agent $i$ 's ex-post equilibrium utility from the facility location $s$ by simply $u_{i}^{*}(s, \boldsymbol{x}, k)$. A useful observation is that the agent's ex-post utilities are (weakly) single-peaked, this result is stated in Proposition 3.2. The proof is straightforward and left to the appendix for the interested reader. Intuitively, the result holds because under the distance-based priority $(\triangleright)$ an agent's priority only (weakly) improves when the facility moves from a location $s<x_{i}$ to a new location $s^{\prime}: s<s^{\prime} \leq x_{i}$ (similarly for $s>x_{i}$ ).

\footnotetext{
${ }^{11}$ In Section 5 we weaken this assumption and consider the problem when the facility can be made excludable.

${ }^{12}$ That is, no agent has an incentive to unilaterally deviate, whatever the preferences of each agent.

${ }^{13}$ This ensure that the binary relation $\triangleright$ is complete.
} 
Proposition 3.2. For any agent $i \in N$ and any pair of instances $\langle\boldsymbol{x}, s, k\rangle$ and $\left\langle\boldsymbol{x}, s^{\prime}, k\right\rangle$, if $s<s^{\prime} \leq$ $x_{i}$ or $x_{i} \leq s^{\prime}<s$ then $u_{i}^{*}(s, \boldsymbol{x}, k) \leq u_{i}^{*}\left(s^{\prime}, \boldsymbol{x}, k\right)$.

In this paper we are interested in 'strategyproof' mechanisms where agents do not have an incentive to misreport their location. In particular, we use the ex-post Dominant-strategy Incentive Compatible (DIC) concept of strategyproofness. That is, a mechanism $M$ is DIC if for every agent $i \in N$

$$
u_{i}^{*}\left(M\left(x_{i}, \hat{\boldsymbol{x}}_{-i}\right), \boldsymbol{x}, k\right) \geq u_{i}^{*}\left(M\left(x_{i}^{\prime}, \hat{\boldsymbol{x}}_{-i}\right), \boldsymbol{x}, k\right)
$$

for every $x_{i}^{\prime}$, for every $\hat{\boldsymbol{x}}_{-i}$, and for every $\boldsymbol{x}_{-i}$. Note that DIC implies that, conditional on the ex-post Nash equilibrium being achieved in the subgame $\Gamma_{x}(s, k)$, the mechanism is dominant-strategy incentive compatible at the reporting stage. Formally speaking, the DIC definition depends on the capacity constraint $k$ however, abusing notation slightly, we omit the $k$ dependence as this will be clear from the context.

Objective of the mechanism designer: In this paper we are interested in DIC mechanisms that perform well with respect to social welfare, i.e., the sum of agents' equilibrium utilities. As is now standard in the algorithmic mechanism design literature we measure the performance of a DIC mechanism by the worst-case approximation ratio.

Given an instance $\langle\boldsymbol{x}, s, k\rangle$, denote the optimal social welfare by $\Pi^{*}(\boldsymbol{x}, k):=\max _{s \in X} \sum_{i=1}^{n} u_{i}^{*}(s, \boldsymbol{x}, k)$, and given a mechanism $M$ let $\Pi_{M}(\boldsymbol{x}, k)$ denote the social welfare attained by the mechanism, i.e.,

$$
\Pi_{M}(\boldsymbol{x}, k):=\sum_{i=1}^{n} u_{i}^{*}(s, \boldsymbol{x}, k) \quad \text { where } s=M(\boldsymbol{x}) .
$$

The mechanism $M$ is an $\alpha$-approximation if

$$
\max _{\boldsymbol{x} \in \prod_{i=1}^{n} X}\left\{\frac{\Pi^{*}(\boldsymbol{x}, k)}{\Pi_{M}(\boldsymbol{x}, k)}\right\} \leq \alpha,
$$

the LHS of (1) is referred to as the approximation ratio. A mechanism (or family of mechanisms) is said to have a lower bound, $\bar{\alpha}$, on the approximation ratio if

$$
\bar{\alpha} \leq \max _{\boldsymbol{x} \in \prod_{i=1}^{n} x}\left\{\frac{\Pi^{*}(\boldsymbol{x}, k)}{\Pi_{M}(\boldsymbol{x}, k)}\right\} .
$$

We refer to a mechanism $M$ that attains the optimal social welfare for all instances $\langle\boldsymbol{x}, s, k\rangle$, and hence is an $\alpha=1$-approximation, as an optimal mechanism. Again, the optimal mechanism definition depends on the capacity constraint $k$ however, abusing notation, we will omit the $k$ dependence as this will be clear from the context. Note that the optimal mechanism need not, and in general will not, be DIC for a given $k$.

REMARK 1. When $k=n$ our model reduces to the well-known facility location problem studied in [Black, 1948, Moulin, 1980, Procaccia and Tennenholtz, 2013]. Accordingly, this case $(k=n)$ is fully resolved: the 'median' mechanism which always locates the facility at the median reported location is both optimal and DIC. However, the case for $k<n$ has not been studied before - this is the focus of the present paper.

To illustrate how the case where $k<n$ differs from the standard $k=n$ setting we provide an example. The example considers a mechanism that is DIC when $k=n$ but for any capacity constraint $k<n$ is not DIC. 
Example 3.3. Let $M$ be the mechanism such that $M(\boldsymbol{x})=\arg \min _{s \in\{1 / 4,3 / 4\}} d\left(s, x_{i}\right)$ for some $i \in N$, tie-breaking in favor of $s=1 / 4$ if necessary. That is, the mechanism locates the facility at either location $1 / 4$ or $3 / 4$ depending on which is closest to agent $i$ 's report.

First, notice that the mechanism $M$ is DIC when $k=n$. If $k=n$ then every agent $i$ is always served by the facility and hence attains utility $1-d\left(s, x_{i}\right)$ for any facility location $s$. It is immediate that agent $i$ can never strictly benefit from misreporting their location.

However, when $k<n$ the mechanism is not DIC. To see this, consider an instance where agent $i$ is located at $3 / 8$ and all other agents are located at $1 / 4$. When agent $i$ truthfully reports, the facility is located at $1 / 4$ and is not served - leading to zero utility. On the other hand, misreporting to $x_{i}^{\prime} \in(1 / 2,1]$ leads to the facility location $3 / 4$ and agent $i$ is the closest agent to the facility. In this case agent $i$ attains strictly higher utility equal to $1-d(3 / 4,3 / 8)>0$. Thus, the mechanism is not DIC for any $k<n$.

\subsection{A complete characterization of DIC mechanisms}

We begin by defining a family of mechanisms called Generalized Median Mechanisms (GMM). This family was introduced by Border and Jordan [Border and Jordan, 1983] for the $k=n$ setting, and provides a partial characterization of DIC mechanisms. The main result of the present paper shows that GMMs provide a complete characterization of mechanisms that are (1) DIC for all $k \leq n$, and (2) DIC for some $k<n$.

Definition 3.4. [Generalized Median Mechanism (GMM)] A mechanism $M$ is said to be a Generalized Median Mechanism (GMM) if for each $S \subseteq N$ there are constants $a_{S}$, such that for all location profiles $\boldsymbol{x}$

$$
M(\boldsymbol{x})=\min _{S \subseteq N} \max \left\{\max _{i \in S}\left\{x_{i}\right\}, a_{S}\right\} .
$$

To build some intuition, we highlight some well-known GMM mechanisms:

(1) The median mechanism ${ }^{14}$ is attained from (3) by setting $a_{S}=1$ for all subsets $S \subseteq N$ with $|S|<\lfloor(n+1) / 2\rfloor$ and $a_{S}=0$ otherwise.

(2) The $s$-constant mechanism ${ }^{15}$ for some location $s \in X$, i.e., the mechanism that always outputs location $s$, is attained from (3) by setting $a_{\emptyset}=s$ and $a_{S}=1$ for all other (nonempty) subsets $S \subseteq N$.

(3) The agent i dictatorship mechanism ${ }^{16}$ is attained from (3) by setting $a_{S}=0$ for $S=\{i\}$ and $a_{S}=1$ for other subsets $S \subseteq N$.

An example of a mechanism that is not a GMM is the dictatorial-style mechanism considered in Example 3.3.

The main result of the present paper is the following characterization: A mechanism $M$ is DIC for some $k<n$ if and only if $M$ is DIC for every $k \leq n$ if and only if $M$ is a GMM. This result is stated in Theorem 3.5.

TheOREM 3.5. Let $M$ be a mechanism. The following are equivalent:

(1) $M$ is a GMM,

(2) $M$ is DIC for some $k<n$,

(3) $M$ is DIC for every $k \leq n$.

\footnotetext{
$\overline{{ }^{14} \mathrm{~A} \text { mechanism }}$ that always outputs the median of the reported location profile i.e., the $\lfloor(n+1) / 2\rfloor$-th smallest report.

${ }^{15}$ A mechanism that always outputs the location $s$.

${ }^{16} \mathrm{~A}$ mechanism that always outputs the location of agent $i$ 's report.
} 
We present the proof via a series of propositions, and utilize a characterization of Border and Jordan Border and Jordan [1983]. Before presenting these propositions we illustrate the contribution of Theorem 3.5, benchmarked against the results of Border and Jordan [1983]: where GMM are shown to be a strict subset of DIC mechanisms when $k=n$. Below, in Figure 2, we present the result of Border and Jordan [1983]. Figure 3 illustrates our contribution. When considering the capacity constrained problem, with $k<n$, the family of DIC mechanisms coincides precisely with the GMM family.

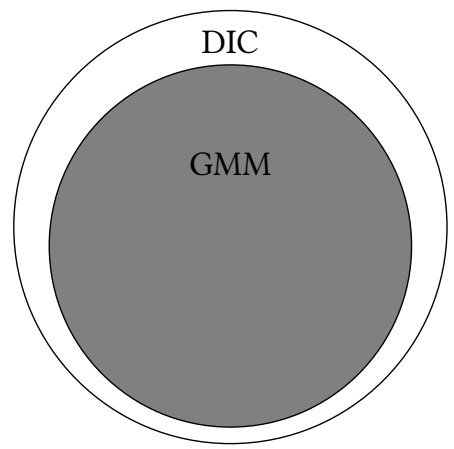

Fig. 2. Setting where $k=n$ [Border and Jordan, 1983].

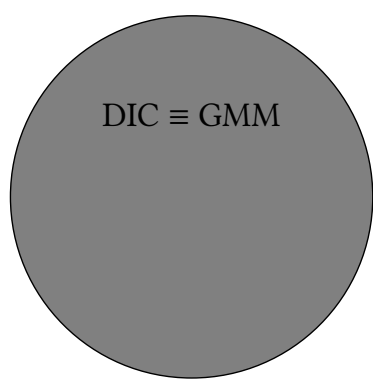

Fig. 3. Setting where $k<n$.

First, we present a result of Border and Jordan [1983] characterizing the family of GMMs via a property of the mechanism that they call 'uncompromising'. Informally speaking, an uncompromising mechanisms means that an agent cannot influence the mechanism output in their favor by reporting extreme locations. The most obvious mechanism satisfying this property is the median mechanism. Formally, a mechanism $M$ is said to be uncompromising if for every profile of locations $\boldsymbol{x}$, and each agent $i \in N$, if $M(\boldsymbol{x})=s$ then

$$
\begin{array}{lll}
x_{i}>s \Longrightarrow M\left(x_{i}^{\prime}, \boldsymbol{x}_{-i}\right)=s & & \text { for all } x_{i}^{\prime} \geq s \\
x_{i}<s \Longrightarrow M\left(x_{i}^{\prime}, \boldsymbol{x}_{-i}\right)=s & \text { for all } x_{i}^{\prime} \leq s . & \text { and, }
\end{array}
$$

Lemma 3.6 (Border AND Jordan [Border And Jordan, 1983]). A mechanism $M$ is uncompromising if and only if it is a GMM.

Note that Lemma 3.6, although proved in the setting where $k=n$, does not rely on any strategic properties of the mechanism and so applies more generally to our setting of interest where $k \leq n$.

We now prove our first proposition towards the characterization result. Proposition 3.7 says that, every GMM is DIC for any $k \leq n$.

Proposition 3.7. Every GMM is DIC for any $k \leq n$.

Proof. Fix $k \leq n$ and let $M$ be a GMM. For the sake of a contradiction suppose that $M$ not DIC. That is, for some agent $i$ with location $x_{i}$, there exist a profile of other agent locations $\boldsymbol{x}_{-i}$, and reports $\hat{\boldsymbol{x}}_{-i}$ such that for some $x_{i}^{\prime} \neq x_{i}$

$$
u_{i}^{*}\left(M\left(x_{i}^{\prime}, \hat{\boldsymbol{x}}_{-i}\right), \boldsymbol{x}, k\right)>u_{i}^{*}\left(M\left(x_{i}, \hat{\boldsymbol{x}}_{-i}\right), \boldsymbol{x}, k\right) .
$$

Define $s^{\prime}=M\left(x_{i}^{\prime}, \hat{\boldsymbol{x}}_{-i}\right)$ and $s=M\left(x_{i}, \hat{\boldsymbol{x}}_{-i}\right)$. It is immediate from (6) that $s \neq x_{i}$ and $s \neq s^{\prime}$. Without loss of generality we assume that $x_{i}>s$. By assumption, $M$ is a GMM and hence by Lemma 3.6 satisfies the uncompromising property. It follows that $x_{i}^{\prime}<s$, since otherwise $x_{i}^{\prime} \geq s$ and (4) would imply $s^{\prime}=s$ contradicting (6). 
Case 1: Suppose $s<s^{\prime}$. Then $x_{i}^{\prime}<s^{\prime}$ and the uncompromising property (5) implies that

$$
M\left(x_{i}^{\prime \prime}, \boldsymbol{x}_{-i}\right)=s^{\prime} \quad \text { for all } x_{i}^{\prime \prime} \leq s^{\prime} .
$$

If $x_{i}^{\prime \prime} \in\left[s, s^{\prime}\right]$ the uncompromising property implies that $M\left(x_{i}^{\prime \prime}, \boldsymbol{x}_{-i}\right)=M\left(x_{i}, \boldsymbol{x}_{-i}\right)$, i.e., $s^{\prime}=s$, which contradicts (6). Thus, we conclude that $x_{i}^{\prime \prime}<s$.

Now consider a new instance where agent $i$ has true location $y_{i}=\varepsilon \in(0, s)$, all other agents have true location $y_{j}=0$ but collectively report $\hat{\boldsymbol{x}}_{-i}$. If agent $i$ reports $y_{i}=\varepsilon$ then the facility location is $s^{\prime}$ and $i$ attains utility $1-d\left(s^{\prime}, \varepsilon\right)$. If instead agent $i$ reports $y_{i}^{\prime}=x_{i}$ then the facility location is $s<s^{\prime}$ and $i$ attains strictly higher utility $1-d(s, \varepsilon)$. Thus, the mechanism is not $k$-DIC - a contradiction.

Case 2: Suppose $s>s^{\prime}$. Since $x_{i}>s>s^{\prime}$, it follows from the single-peaked property (Proposition 3.2) that $u_{i}^{*}(s, \boldsymbol{x}, k) \geq u_{i}^{*}\left(s^{\prime}, \boldsymbol{x}, k\right)$. This contradicts (6).

We now prove our second proposition towards the characterization result. Proposition 3.8 says that, if a mechanism is DIC for some $k<n$ then it is DIC for $k=n$. Thus, the DIC requirement is more restrictive for $k<n$ than for $k=n$ - meaning that the capacity constraints induce new strategic concerns for the mechanism designer.

Proposition 3.8. If a mechanism M is DIC, for some $k<n$, then it is DIC for $k=n$. The converse is not true.

Proof. We prove the contrapositive. Suppose that $M$ is not DIC for $k=n$. That is, for some agent $i$ with location $x_{i}$ there exists a report $x_{i}^{\prime}$, a profile of other agent reports $\hat{\boldsymbol{x}}_{-i}$, and a profile of other agent locations $\boldsymbol{x}_{-i}$ such that

$$
u_{i}^{*}\left(M\left(x_{i}^{\prime}, \hat{\boldsymbol{x}}_{-i}\right), \boldsymbol{x}, n\right)>u_{i}^{*}\left(M\left(x_{i}, \hat{\boldsymbol{x}}_{-i}\right), \boldsymbol{x}, n\right) .
$$

Let $s^{\prime}=M\left(x_{i}^{\prime}, \hat{\boldsymbol{x}}_{-i}\right)$ and $s=M\left(x_{i}, \hat{\boldsymbol{x}}_{-i}\right)$. When $k=n$ all agents are served and so (7) simplifies to

$$
1-d\left(s^{\prime}, x_{i}\right)>1-d\left(s, x_{i}\right) \text {. }
$$

Now we consider the same profile of reports but for an arbitrary $k<n$. Furthermore, suppose all agents have location equal to $x_{i}$ and agent $i$ has highest priority ( $\triangleright$ ), i.e., after tie-breaking. The mechanism output is independent of agent true locations and so we still attain $M\left(x_{i}^{\prime}, \hat{\boldsymbol{x}}_{-i}\right)=s^{\prime}$ and $M\left(x_{i}^{\prime}, \hat{\boldsymbol{x}}_{-i}\right)=s$. Furthermore, since $i$ has highest priority (recall that the priority is distance-based but in this instance all agents are equidistant for every facility location) they are always served for every facility location. In particular, the utility from reporting truthfully is $1-d\left(s, x_{i}\right)$ and misreporting is $1-d\left(s^{\prime}, x_{i}\right)$ - the latter provides strictly higher utility, as per (8). We conclude that the mechanism is not DIC, and since $k<n$ was chosen arbitrarily it holds for all $k<n$.

The final statement in the proposition was shown in Example 3.3.

We now prove our third and final proposition, which completes the characterization result. Proposition 3.9 says that, if a mechanism is DIC for some $k<n$ then it is a GMM.

Proposition 3.9. If a mechanism $M$ is DIC, for some $k<n$, then it is a GMM.

Proof. Let $M$ be a mechanism that is DIC for some $k<n$.

First, consider an instance where an arbitrary agent $i$ has location $x_{i}$, and the other agents report $\hat{\boldsymbol{x}}_{-i}$. If $i$ reports truthfully the mechanism outputs some location that we denote as $s$, i.e.,

$$
s:=M\left(x_{i}, \hat{\boldsymbol{x}}_{-i}\right) \text {. }
$$

If $s=x_{i}$ then consider an alternate location and profile of other agents' reports so that the equality does not hold. If no such location and report profile exists then the mechanism always coincides 
with agent $i$ 's report; that is, the mechanism is the agent $i$ dictatorship mechanism, which is a GMM.

Now suppose $s \neq x_{i}$, and without loss of generality assume $s<x_{i}$. By assumption $M$ is DIC, for some $k<n$, and so it must be that for all $x_{i}^{\prime}$

$$
u_{i}^{*}(s, x, k) \geq u_{i}^{*}\left(M\left(x_{i}^{\prime}, \hat{x}_{-i}\right), x, k\right),
$$

where $\boldsymbol{x}$ denotes the location profile of all agents.

We now show that deviations by agent $i$ satisfy the uncompromising property, i.e., for any $x_{i}^{\prime} \geq s$ $M\left(x_{i}^{\prime}, \hat{\boldsymbol{x}}_{-i}\right)=s$. To do so, we analyze different cases and sequential refine the possible values of $M\left(x_{i}^{\prime}, \hat{\boldsymbol{x}}_{-i}\right)$, we the derive a contradiction to eventually conclude that $M\left(x_{i}^{\prime}, \hat{\boldsymbol{x}}_{-i}\right)=s$.

Case 1: Suppose all other agents have location $s$. When agent $i$ truthfully reports $x_{i}$ the facility location is $s$ and they attain zero utility. Now consider some report $x_{i}^{\prime} \geq s$, leading to facility location

$$
s_{x_{i}^{\prime}}:=M\left(x_{i}^{\prime}, \hat{\boldsymbol{x}}_{-i}\right) .
$$

If $s_{x_{i}^{\prime}} \in\left(\frac{s+x_{i}}{2}, 1\right]$ for any $x_{i}^{\prime} \geq s$ we attain a contradiction, since this agent $i$ would be served from this report and attain strictly more utility than being truthful. We conclude that

$$
s_{x_{i}^{\prime}} \in[0, s) \cup\{s\} \cup\left(s, \frac{s+x_{i}}{2}\right) \quad \text { for all } x_{i}^{\prime} \geq s .
$$

Case 2: Suppose all other agents have location 1, noting that $s<x_{i} \leq 1$. In the event that $x_{i}=1$ (in which case all agents are equidistant from every facility location), assume agent $i$ has the highest priority in the tie-breaking rule $(\triangleright)$. When agent $i$ truthfully reports their location they are served and attain utility $1-d\left(s, x_{i}\right)$. To avoid a contradiction of (10), it must be that $s_{x_{i}^{\prime}} \leq s$. Thus, we conclude

$$
s_{x_{i}^{\prime}} \in[0, s) \cup\{s\} \quad \text { for all } x_{i}^{\prime} \geq s .
$$

For the sake of a contradiction suppose there exists some $x_{i}^{\prime \prime} \geq s$ such that

$$
s_{x_{i}^{\prime \prime}} \in[0, s) .
$$

Consider a new instance where agent $i$ 's location is $y_{i}=x_{i}^{\prime \prime}$ (note that $x_{i}^{\prime \prime} \geq s$ ), all other agents have location 1 , and the other agents report $\hat{\boldsymbol{x}}_{-i}$ (the same profile of reports as per (10)). In the event that $y_{i}=x_{i}^{\prime \prime}=1$ (in which case all agents are equidistant from every facility location), assume agent $i$ has the highest priority in the tie-breaking rule ( $\triangleright)$. If agent $i$ reports their location $y_{i}$ the facility location is $s_{y_{i}}=s_{x_{i}^{\prime \prime}}<s$, as per (11), and they attain utility $1-d\left(s_{y_{i}}, y_{i}\right)$. But now misreporting to $y_{i}^{\prime}=x_{i}$ then as per (9) the facility location is $s$ where

$$
s_{y_{i}}<s \leq y_{i}
$$

leading to utility $1-d\left(s, y_{i}\right)$. This is a contradiction of the mechanism being DIC, since $d\left(s, y_{i}\right)<$ $d\left(s_{y_{i}}, y_{i}\right)$; that is, agent $i$ by reporting $y_{i}^{\prime}$ instead of their true location $y_{i}$ attains strictly higher utility. We conclude that $s_{x_{i}^{\prime}}=s$ for all $x_{i}^{\prime} \geq s$. Thus, the mechanism is uncompromising and hence a GMM.

\section{APPROXIMATION OF DIC MECHANISMS}

Given the characterization result (Theorem 3.5) of the previous section, there is no distinction between the family of mechanisms that are DIC for some $k<n$, and the family of mechanisms that are DIC for all $k \leq n$ : both families are equal to the GMM family. Accordingly, we will now simply refer to a mechanism as being DIC. 


\subsection{Optimal mechanism is not DIC}

We first show that, in general for $k<n$, the optimal mechanism is not DIC. Note that this result contrasts with the $k=n$ setting where the median mechanism is both optimal and DIC (Remark 1).

Theorem 4.1. The optimal mechanism is DIC if and only if $k \in\{1, n\}$.

Proof. The backward direction of the theorem statement is straightforward: If $k=1$ then for any $i \in N$ the agent $i$ dictator mechanism, where the mechanism output always coincides with agent $i$ 's report, is both optimal and DIC. This is trivial and we do not provide further details. If $k=n$ then the median mechanism is both optimal and DIC. This result has long been known and can be found in Black [1948], Moulin [1980], Procaccia and Tennenholtz [2013].

We now prove the forward direction using the contrapositive. Let $k \notin\{1, n\}$ and partition the agent into $\lfloor n / k\rfloor$ groups of size $k$, denoted by $N_{t}$ for $t=1,2, \ldots,\lfloor n / k\rfloor$, and one group of size $n-\lfloor n / k\rfloor$, denoted by $N_{\lfloor n / k\rfloor+1}$. We now identify $\lfloor n / k\rfloor+1$ locations in $[0,1]$, let

$$
y_{t}=\frac{t}{\lfloor n / k\rfloor+1} \quad \text { for } t=1,2, \ldots,\lfloor n / k\rfloor+1 .
$$

Consider a scenario such that for each $t=1,2, \ldots,\lfloor n / k\rfloor+1$, all but one agent in $N_{t}$ is located at $y_{t}$ and a single agent is located at $y_{t}-t \varepsilon$ for some sufficiently small $\varepsilon>0$. In each instance denote the single agent located at $y_{t}-t \varepsilon$ by $i_{t} \in N_{t}$.

In this scenario it is immediate the optimal welfare is attained by locating the facility at location $y_{1}$, leading to a social welfare of $k-\varepsilon$ and agent $i_{1}$ attain utility $1-\varepsilon$.

Now in a new scenario where agent $i_{1}$ is located at $y_{1}-3 \varepsilon$ the optimal mechanism must locate the facility at $y_{2}$. In this case agent $i_{1}$ attains utility zero. However, if agent $i_{1}$ misreport their location to $y_{1}-\varepsilon$ then (as shown above) the facility location will be $y_{1}$ and they will attain strictly higher utility $1-\varepsilon$. That is, the optimal mechanism is not DIC for $k \notin\{1, n\}$.

Despite Theorem 4.1 stating a stark impossibility result, we note that absent strategic manipulations by the agents the optimal mechanism can be efficiently computed. Remark 2 says that, for any $k \leq n$ the optimal mechanism's output and corresponding welfare can be computed in polynomial time.

REMARK 2. The optimal facility location and welfare can be computed in polynomial time for any $k \leq n$.

We sketch an informal argument for Remark 2. Order the agents $i \in N$ such that $x_{i} \leq x_{j}$ if and only if $i \leq j$. It is straightforward to show that an optimal solution has two features (1) the facility serves a contiguous set of $k$ agents, i.e., if agent $i$ and $i+2$ are served then agent $i+1$ is served, and (2) the facility is located at the median of these $k$ served agents. Given these features, it is immediate that a polynomial-time procedure exists by simply comparing the welfare produced by, the at most $n$, sets of $k$ contiguous agents.

\subsection{Lower bound on DIC approximation}

Utilizing the characterization result of DIC mechanisms via the family of GMMs, we provide a lower bound on the approximation ratio for all DIC mechanisms.

Theorem 4.2 shows that at best a DIC mechanisms provides a $2 \frac{k}{k+1}$-approximation when $k \leq$ $\lceil(n-1) / 2\rceil$, and otherwise provides at best an $\max \left\{\frac{n-1}{k+1}, 1\right\}$-approximation. This lower bound on the approximation ratio is illustrated in Figure 1.

Theorem 4.2. Let $n \geq 2$. A DIC mechanism is at best an $\alpha$-approximation with $\alpha=2 \frac{k}{k+1}$ when $1 \leq k \leq\lceil(n-1) / 2\rceil$, and $\alpha=\max \left\{\frac{n-1}{k+1}, 1\right\}$ otherwise. 
Proof. Let $M$ be a DIC mechanism, and consider a scenario where all $n$ agents have distinct locations contained in the interval $I=(1 / 2-1 / 2 \varepsilon, 1 / 2+1 / 2 \varepsilon)$ for some sufficiently small $\varepsilon>0$. Denote the profile of agent locations by $\boldsymbol{x}$, and the mechanism's corresponding output by $s=M(\boldsymbol{x})$.

We consider two cases.

Case 1: Suppose $s \notin I$ and without loss of generality assume $s<1 / 2-1 / 2 \varepsilon$. Now suppose that sequentially agents $i=1,2, \ldots, n$ have their locations changed and kept at $x_{i}=1$, and consider the sequence of facility locations produced by the mechanism $s_{1}, s_{2}, \ldots, s_{n}$. By the uncompromising property (satisfied by $M$ since it is a GMM) the location of the facility never changes from $s$. That is, $s_{n}=s$ despite every agent having location at 1 . The optimal social welfare in this scenario is clearly $k$, however, the mechanism provides welfare of

$$
k(1-d(s, 1))=k s<k(1 / 2-1 / 2 \varepsilon) \rightarrow k / 2 \quad \text { as } \varepsilon \rightarrow 0 .
$$

Thus, the approximation ratio is at best $k /(k / 2)=2$.

Case 2: Suppose $s \in I$ and without loss of generality assume $s \leq 1 / 2$. Let $\lambda_{1}, \lambda_{2}$ be the number of agents with locations strictly less than $s$, and strictly above $s$, respectively. Note that $\lambda_{1}+\lambda_{2} \in$ $\{n-1, n\}$. Similar to Case 1 , suppose the $\lambda_{1}$ agents instead had location at 0 and the $\lambda_{2}$ agents had location at 1 - by the uncompromising property the facility location is unchanged.

To attain the bound on the approximation ratio we consider two subcases where $k \leq\lceil(n-1) / 2\rceil$ and $k>\lceil(n-1) / 2\rceil$.

In the first subcase $(k \leq\lceil(n-1) / 2\rceil)$ : the optimal welfare is $k$, since either $\lambda_{1}$ or $\lambda_{2}$ exceeds $k$ meaning that $k$ agents can be served at either 0 or 1 . The mechanism's welfare is at most

$$
1+(k-1)(1-d(s, 0))<1+(k-1)(1 / 2-1 / 2 \varepsilon) \rightarrow 1 / 2+k / 2 \quad \text { as } \varepsilon \rightarrow 0 .
$$

Thus, the approximation ratio is at best $k /(1 / 2+k / 2)=2 k /(k+1)$.

In the second subcase $(k>\lceil(n-1) / 2\rceil)$ : the optimal welfare is at worst $\lceil(n-1) / 2\rceil$, i.e., when the facility serves either $\lambda_{1}$ or $\lambda_{2}$ agents (whichever is larger) from location 0 or 1 . The mechanism's welfare is at most

$$
1+(k-1)(1-d(0, s))<k-(k-1)(1 / 2-1 / 2 \varepsilon) \rightarrow k / 2+1 / 2 \quad \text { as } \varepsilon \rightarrow 0 .
$$

Thus, the approximation ratio is at best $\lceil(n-1) / 2\rceil /(k / 2+1 / 2)$, but

$$
\lceil(n-1) / 2\rceil /(k / 2+1 / 2) \geq \frac{(n-1) / 2}{(k+1) / 2}=\frac{n-1}{k+1} .
$$

Furthermore since $k>(n-1) / 2$ it follows that $\frac{n-1}{k+1}<2$. Of course, this bound is only meaningful when $n-1 / k+1>1$.

We conclude that when $k \leq\lceil(n-1) / 2\rceil$ the approximation ratio is at best $2 \frac{k}{k+1}$ and otherwise is at best $\max \left\{\frac{n-1}{k+1}, 1\right\}$.

\subsection{Optimized approximation ratio for DIC Mechanism}

We now analyze the performance of the median mechanism for general $k \leq n$. In instances where $k \in\{1, n\}$, the median mechanism is both optimal mechanism and DIC (Theorem 4.1). Furthermore, this mechanism is DIC for all $k \leq n$ since the median mechanism is a GMM (Theorem 3.5).

Theorem 4.3 says that the median mechanism is an $\alpha$-approximation where $\alpha=2 \frac{k}{k+1}$ when $k \leq\lfloor(n+1) / 2\rfloor$, and $\alpha=\min \left\{2 \frac{k}{k+1}, 1+2 \frac{n-k+1}{3 k-2 n-2}\right\}$ otherwise. In particular, this means that the median mechanism is optimal among DIC mechanism for $k \leq\lfloor(n-1) / 2\rfloor$ since the approximationratio matches the lower bound found in Theorem 4.2. These approximation results are illustrated in Figure 1. 
Theorem 4.3. Let $n \geq 5$. The median mechanism is an $\alpha$-approximation with $\alpha=2 \frac{k}{k+1}$ for $k \leq\lfloor(n+1) / 2\rfloor$, and $\alpha=\min \left\{2 \frac{k}{k+1}, 1+2 \frac{n-k+1}{3 k-2 n-2}\right\}$ otherwise.

Proof. Let $n \geq 5$. Throughout the proof let $i_{m}$ denote the agent with median location (choose the agent arbitrarily if multiple such agents exist), and let $s_{m}$ denote the median location. The median mechanism provides welfare

$$
\Pi_{M}(\boldsymbol{x}, k)=\max _{N^{\prime} \in N_{k}} \sum_{i \in N_{k}}\left(1-d\left(s_{m}, x_{i}\right)\right)=1+\max _{N^{\prime} \in N_{k-1, i m}} \sum_{i \in N_{k-1, i_{m}}}\left(1-d\left(s_{m}, x_{i}\right)\right)
$$

where $N_{k}$ is the set of all $k$-sized subsets of $N$ and $N_{k-1, i_{m}}$ is the set of all $(k-1)$-sized subsets of $N \backslash\left\{i_{m}\right\}$. This follows since the subset of agents served are always the $k$-closest to the facility location. Hence, given a facility location, the served subset is welfare maximizing. Furthermore, the median location coincides with at least one agent's location, i.e., agent $i_{m}$.

First, we provide an upper bound on the approximation-ratio for all $k$. The median mechanism locates the facility at the $\lfloor(n+1) / 2\rfloor$-th location and hence there are $\lfloor(n+1) / 2\rfloor-1$ agents with locations (weakly) below and $\lceil(n+1) / 2\rceil-1$ with locations (strictly) above. A lower bound on the median mechanism's welfare is attained when the agents below and above the median location at located at 0 and 1 , respectively. Thus,

$$
\Pi_{M}(\boldsymbol{x}, k) \geq 1+(k-1) \max \left\{1-d\left(s_{m}, 0\right), 1-d\left(s_{m}, 1\right)\right\}
$$

and since either $d\left(s_{m}, 0\right) \leq 1 / 2$ or $d\left(s_{m}, 1\right) \leq 1 / 2$ it follows that $\Pi_{M}(\boldsymbol{x}, k) \geq(k+1) / 2$. This leads to an upper bound on the approximation-ratio of $k /((k+1) / 2)=2 \frac{k}{k+1}$ for all $k$, since the optimal welfare is always bounded above by $k$.

Now we attain a tighter upper bound for certain values of $k$. To do so, we bound the median welfare using the optimal welfare. Let $s^{*}$ be the location of the facility under the optimal mechanism. Let $N_{m}^{*}$ denote the set of $k$ agents served under the median mechanism, and let $N^{*}$ denote the set of $k$ agents served under the optimal mechanism. We have

$$
\begin{aligned}
\Pi_{M}(\boldsymbol{x}, k) & \geq \sum_{i \in N^{*}}\left(1-d\left(s_{m}, x_{i}\right)\right) \\
& =\sum_{i \in N^{*}}\left(1-d\left(s_{m}, x_{i}\right)-d\left(s^{*}, x_{i}\right)+d\left(s^{*}, x_{i}\right)\right) \\
& =\Pi^{*}(\boldsymbol{x}, k)-\sum_{i \in N^{*}}\left(d\left(s_{m}, x_{i}\right)-d\left(s^{*}, x_{i}\right)\right) .
\end{aligned}
$$

Clearly, the lower bound is smallest when $s_{m} \neq s^{*}$, without loss of generality assume that $s_{m}<s^{*}$. Let $N_{1}^{*}, N_{2}^{*}$ be a partition of $N^{*}$ such that $\left|N_{1}^{*}\right|,\left|N_{2}^{*}\right| \leq\lfloor(n+1) / 2\rfloor$ and all agents in $N_{1}^{*}$ have location in $\left[0, s_{m}\right]$ and agent in $N_{2}^{*}$ have location in $\left[s_{m}, 1\right]$. Such a partition of $N^{*}$ exists since the location $s_{m}$ coincides with the $\lfloor(n+1) / 2\rfloor$ highest location. Using this partition we further bound the median 
mechanism's welfare:

$$
\begin{aligned}
\Pi_{M}(\boldsymbol{x}, k) & \geq \Pi^{*}(\boldsymbol{x}, k)-\sum_{i \in N_{1}^{*}}\left(d\left(s_{m}, x_{i}\right)-d\left(s^{*}, x_{i}\right)\right)-\sum_{i \in N_{2}^{*}}\left(d\left(s_{m}, x_{i}\right)-d\left(s^{*}, x_{i}\right)\right) \\
& \geq \Pi^{*}(\boldsymbol{x}, k)-\left|N_{1}^{*}\right| \max _{x \in\left[0, s_{m}\right]}\left(s_{m}-s^{*}-2 x\right)-\left|N_{2}^{*}\right| \max _{x \in\left[s_{m}, 1\right]}\left(x_{i}-s_{m}-\left|s^{*}-x_{i}\right|\right) \\
& \geq \Pi^{*}(\boldsymbol{x}, k)-\left|N_{1}^{*}\right|\left(s_{m}-s^{*}\right)-\left|N_{2}^{*}\right|\left(s^{*}-s_{m}\right) \\
& \geq \Pi^{*}(\boldsymbol{x}, k)-\left(\left|N_{2}^{*}\right|-\left|N_{1}^{*}\right|\right)\left(s^{*}-s_{m}\right) \\
& \geq \Pi^{*}(\boldsymbol{x}, k)-\left(\left|N_{2}^{*}\right|-\left|N_{1}^{*}\right|\right) .
\end{aligned}
$$

We now attain our lower bound by considering the maximum value of $\left|N_{2}^{*}\right|-\left|N_{1}^{*}\right|$. For $k \leq$ $\lfloor(n+1) / 2\rfloor$, the value can only be guaranteed to be no larger than $k$ - leading to a trivial zero lower on $\Pi_{M}(\boldsymbol{x}, k)$. However, for $k>\lfloor(n+1) / 2\rfloor$ we attain a more useful bound by noting that

$$
\left(\left|N_{2}^{*}\right|-\left|N_{1}^{*}\right|\right) \leq\lfloor(n+1) / 2\rfloor-(k-\lfloor(n+1) / 2\rfloor)=2\lfloor(n+1) / 2\rfloor-k \leq n+1-k .
$$

This lead to an approximation-ratio upper bound of

$$
\max _{\boldsymbol{x} \in \prod_{i=1}^{n} X}\left\{\frac{\Pi^{*}(\boldsymbol{x}, k)}{\Pi^{*}(\boldsymbol{x}, k)-n-1+k}\right\}=\max _{\boldsymbol{x} \in \prod_{i=1}^{n} X}\left\{1+\frac{n+1-k}{\Pi^{*}(\boldsymbol{x}, k)-n-1+k}\right\} .
$$

Furthermore, for any instance $\Pi^{*}(\boldsymbol{x}, k) \geq k / 2$ since at least as much welfare is attained by locating the facility at $s=1 / 2$. Thus, an upper bound on the approximation ratio is

$$
1+\frac{n+1-k}{k / 2-n-1+k}=1+2 \frac{n+1-k}{3 k-2 n-2} .
$$

\section{EXTENSION: LOCATION-ALLOCATION MECHANISMS}

In this section we consider an extension of our framework where the mechanism designer is able to dictate which agents are served by the facility. Note that this extension introduces an underlying assumption that the facility is excludable. In practice, a designer may be able to dictate which agents are served by issuing permits or, when costs are not prohibitive, checking the identities of agents attempting to benefit from the facility.

Previously, a mechanism $M: \prod_{i \in N} X \rightarrow X$ was defined as a function mapping a profile of locations to a single facility location. In our extension, a mechanism not only locates the facility but also chooses a subset of at most $k$ agents to be served by the facility, if they so choose. We denote these extended mechanisms by

$$
M_{A}: \prod_{i \in N} X \rightarrow X \times N_{k}
$$

where $N_{k}=\{A \subseteq N: 0<|A| \leq k\}$. We call these mechanisms location-allocation mechanisms, to distinguish them from the (location-only) mechanisms considered in earlier sections of the present paper. The output of the mechanism is a pair $(s, A) \in X \times N_{k}$ where $s \in X$ denotes the facility location and $A \in N_{k}$ denotes the subset of agents allocated to the facility. Abusing notation slightly we will denote the mechanism output from a location profile $\boldsymbol{x}$ by $s_{\boldsymbol{x}}$ and $A_{\boldsymbol{x}}$ where $M_{A}(\boldsymbol{x})=$ $\left(s_{\boldsymbol{x}}, A_{\boldsymbol{x}}\right)$. An agent $i \in A$ is guaranteed to be served by the facility if they so choose, whilst an agent $i \notin A$ is never served.

We omit the details, but it is immediate that the modified subgame $\Gamma_{\boldsymbol{x}}(s, k, A)$ has an essentially unique ex-post Nash equilibrium where all agents $i \in A$ are served by the facility and the remaining agents are not. Thus, we assume that agent $i$ reports their location to the mechanism designer with 
the understanding that they will be served by the facility if and only if $i \in A$, as per the ex-post Nash equilibrium. Note that the strategyproof concept, DIC, in this section still coincides with the concept used in the earlier sections, albeit with the modified subgame explained above.

We first remark that the revelation principle [Gibbard, 1973] does not apply. A location-only mechanism, based on the profile of agent reports, $\hat{\boldsymbol{x}}$, outputs a facility location $s-$ that depends on $\hat{\boldsymbol{x}}$ - and a subset of $k$ agents are then allocated to the facility, via the ex-post Nash equilibrium, $A \subseteq N$ - this subset depends on the agent true locations $\boldsymbol{x}$ and not the reports $\hat{\boldsymbol{x}}$. In contrast, a locationallocation mechanism outputs both a facility location and an allocation of $\leq k$ agents to the facility depending on agent reports $\hat{\boldsymbol{x}}$, and not true locations $\boldsymbol{x}$. Thus, an agent misreporting their location - in a way that does not affect the facility location - will never affect whether or not they are served by the facility under a location-only mechanisms. However, under a location-allocation mechanism the agent may potentially benefit from the misreport if they are now allocated to the facility by the mechanism.

We now show that no 'reasonable' location-allocation-mechanism is DIC. In particular, we only enforce one criteria, which is a weak form of anonymity. Informally speaking, we require that the location-allocation mechanism allocates agents to the facility independently of their label if their report is distinct from all other agents. The usual definition of anonymity is not directly applicable since with a deterministic mechanism, if all agents report identical locations the mechanism must discriminate against at least $n-k$ agents who will not be included in the allocation set $A$.

To formally define our anonymity condition we first introduce the notion of an $i$-identifiable location profile. This is simply a profile where agent $i$ is uniquely identified by their report.

Definition 5.1 (i-identifiable location profile). Let $i \in N$. A location profile $\boldsymbol{x}$ is $i$-identifiable if

$$
x_{i} \neq x_{j} \quad \text { for all } j \in N \backslash\{i\} .
$$

We now define our anonymity condition, which we call allocation-anonymous since the condition only applies to the allocation set rather than the facility location. Informally speaking, the allocation-anonymous condition requires that for every $i$-identifiable location profile, whether or not agent $i$ is allocated to the facility does not depend on $i$ 's label. Given that allocation-anonymity only applies to $i$-identifiable location profiles the condition is relatively weak.

Definition 5.2 (Allocation-anonymous). The mechanism $M_{A}$ is said to be allocation-anonymous if for every distinct $i, j \in N$ and every $i$-identifiable location profile $\boldsymbol{x}$, the modified profile $\boldsymbol{x}^{\prime}$ such that $x_{\ell}=x_{\ell}^{\prime}$ for all $\ell \neq i, j$ and

$$
x_{i}^{\prime}=x_{j} \quad \text { and } \quad x_{j}^{\prime}=x_{i},
$$

we have

$$
i \in A_{\boldsymbol{x}} \Longleftrightarrow j \in A_{\boldsymbol{x}^{\prime}}
$$

where $A_{\boldsymbol{x}}$ is such that $M_{A}(\boldsymbol{x})=\left(s_{\boldsymbol{x}}, A_{\boldsymbol{x}}\right)$.

We now show that if we restrict our attention to allocation-anonymous mechanisms there is no DIC location-allocation mechanism.

THEOREM 5.3. Let $k<n$, any location-allocation mechanism $M_{A}$ that is allocation-anonymous is not DIC.

Proof. For the sake of a contradiction suppose that $M_{A}$ is a location-allocation mechanism that is both allocation-anonymous and DIC.

First consider a location profile $x$ where $x_{i}=3 / 4$ for all $i \in N$, and denote the output of the mechanism by $M_{A}(\boldsymbol{x})=(s, A)$. Let $i^{*}$ be some agent such that $i^{*} \in A$ and $j^{*}$ some agent such that $j^{*} \notin A$. In this outcome agent $j^{*}$ attains utility zero, since $j^{*} \notin A$. 
Now consider another location profile $\boldsymbol{x}^{\prime}$ such that $x_{i}^{\prime}=3 / 4$ for all $i \in N \backslash\left\{j^{*}\right\}$ and $x_{j^{*}}^{\prime}=1 / 2$. Note that the profile $\boldsymbol{x}^{\prime}$ can be achieved via a unilateral deviation from the profile $\boldsymbol{x}$ by agent $j^{*}$. Denote the mechanism's output from this location profile by $M_{A}\left(\boldsymbol{x}^{\prime}\right)=\left(s^{\prime}, A^{\prime}\right)$. We consider two cases and derive a contradiction in each case.

Case 1: Suppose $j^{*} \in A^{\prime}$ and suppose that $x$ is the true location of all agents. In this case agent $j^{*}$ by misreporting their location to $x_{j^{*}}^{\prime}=1 / 2$ strictly profits, since under the profile $\boldsymbol{x}^{\prime}$ we have $j^{*} \in A^{\prime}$ and their utility is now $1-d\left(s^{\prime}, \frac{3}{4}\right)>0$ rather than zero. Thus, we have a contradiction.

Case 2: Suppose $j^{*} \notin A^{\prime}$ and suppose that the agents have true locations $y_{\ell}=3 / 4$ for all $\ell \in N \backslash\left\{i^{*}\right\}$ and $y_{i^{*}}=1 / 2$. Denote the mechanism's output from location profile $\boldsymbol{y}$ by $M_{A}\left(\boldsymbol{x}^{\prime}\right)=$ $\left(s^{\prime \prime}, A^{\prime \prime}\right)$. Notice that $\boldsymbol{x}^{\prime}$ is a $j^{*}$-identifiable location profile and the profile $\boldsymbol{y}$ satisfies the condition in Definition 5.2, and so by the allocation-anonymous property we require that

$$
i^{*} \in A^{\prime \prime} \Longleftrightarrow j^{*} \in A^{\prime} \text {. }
$$

Thus, we infer that $i^{*} \notin A^{\prime \prime}$ and attain zero utility under the location profile $\boldsymbol{y}$. Now suppose agent $i^{*}$ unilaterally deviates and reports the location $y_{i^{*}}^{\prime}=3 / 4$. In this case, the location profile coincides with the profile $\boldsymbol{x}$ where $x_{\ell}=3 / 4$ for all $\ell \in N$. But recall that $M_{A}(\boldsymbol{x})=(s, A)$ and $i^{*}$ was taken to be some agent such that $i^{*} \in A$. Thus, under this unilaterally misreport agent $i^{*}$ is now served and attain strictly positive utility of $1-d\left(s, y_{i^{*}}\right)>0$. This is a profitable deviation and contradicts our assumption that the mechanism $M_{A}$ was DIC. We conclude that there is no location-allocation mechanism that is both allocation-anonymous and DIC.

The above impossibility result means that the extensive-form approach taken in the main body of this paper is crucial for DIC mechanisms that are non-dictatorial. The use of an extensive-form game and corresponding ex-post Nash equilibria to decide the allocation of agents to the facility reduces the incentive compatibility constraints faced by the mechanism designer. Furthermore, the result suggests that the excludability of the facility presents a greater challenge for incentive compatibility than rivalry.

\section{DISCUSSION AND CONCLUSION}

We now conclude the paper with a brief discussion of future research directions.

Extensions to multiple facilities: In the present paper we focused on the case of a single capacity constrained facility location problem. Extending the capacity constrained to multiple facilities presents a number of challenges. Firstly, the subgame induced from a profile of facility location will lead to multiple equilibria that are not welfare (nor utility) equivalent. Furthermore, even when ignoring the multiplicity of equilibria issues, the mechanism design problem is drastically more complicated - as is the algorithmic problem of finding the optimal facility locations (see Brimberg et al. [Brimberg et al., 2001]). A recent contribution by Golowich, Narasimhan and Parkes [Golowich et al., 2018] explores the mechanism design problem for multiple facilities without capacity constraints.

Weakening DIC: A natural direction to consider is weakening the strategproofness concept (DIC) that we use in the present paper. The DIC requirement is very strong: agents must attain maximal ex-post utility from reporting their location no matter what other agents report, and other agents' true locations. The weaker notion of ex-post Incentive Compatible (IC) may be interesting to be explore for both characterization and performance results. This notion requires that agents 
attain maximal ex-post utility from reporting their location no matter the other agents' true locations, but conditional on the other agents reporting truthfully. It is straightforward to construct IC mechanisms that out-perform the median mechanism for certain parameter ranges.

Conclusion: In this paper we initiated the study of the capacity constrained facility location problem from a mechanism design perspective. We formalized a model that allows the subset of served agents to be endogenously derived from equilibrium outcomes. Our main contribution is a complete characterization of all DIC mechanisms via the family of GMM mechanisms. This characterization also provides a novel perspective to an open problem in regard to GMM mechanisms, posed in [Border and Jordan, 1983]. Our second contribution is an analysis of the performance of DIC mechanisms with respect to social welfare - where we also show that the well-known median mechanism is optimal among DIC mechanism for certain parameter ranges. Finally, we show that extending the space of mechanisms to allow the mechanism to allocate agents to the facility leads to a stark impossibility result. Namely, there is no allocation-anonymous DIC mechanism which both locates the facility and stipulates the subset of agents to be served.

\section{REFERENCES}

A. Abdulkadiroğlu and T. Sönmez. 2003. School Choice: A mechanism Design Approach. American Economic Review 93, 3 (2003), 729-747.

I. Ashlagi and A. Roth. 2011. Individual rationality and participation in large scale, multi-hospital kidney exchange. In Proceedings of the 12th ACM Conference on Electronic Commerce (ACM-EC). ACM Press, 321-322.

S. Barbarà, J. Massó, and S. Serizawa. 1998. Strategy-proof voting on compact ranges. Games and Economic Behavior 25 (1998), 272-291.

D. Black. 1948. On the rationale of group decision-making. fournal of Political Economy 56, 1 (1948), 23-34.

K. C. Border and J. S. Jordan. 1983. Straightforward Elections, Unanimity and Phantom Voters. The Review of Economic Studies 50, 1 (1983), 153-170.

M. L. Brandeau and S. S. Chiu. 1989. An overview of representative problems in location research. Management Science 35, 6 (1989), 645-674.

J. Brimberg, E. Korach, M. Eben-Chaim, and A. Mehrez. 2001. The capacitated p-facility location problem on the real line. International Transactions in Operational Research 8 (2001), 727-738.

M. Charikar, S. Guha, E. Tardos, and D.B. Shmoys. 2002. A constant-factor approximation algorithm for the k-median problem. J. Comput. System Sci. 65, 1 (2002), 129-149.

S. Ching. 1997. Strategy-proofness and “Median Voters”. International fournal of Game Theory 26 (1997), 473-490.

M. Cygan, M. T. Hajiaghayi, and S. Khuller. 2012. LP rounding for k-centers with non-uniform hard capacities. In Foundations of Computer Science (FOCS), 2012 IEEE 53rd Annual Symposium on. IEEE, 273-282.

M. Feldman, A. Fiat, and I. Golomb. 2016. On voting and facility location. In Proceedings of the 17th ACM Conference on Electronic Commerce (ACM-EC). ACM Press, 269-286.

A. Gibbard. 1973. Manipulation of voting schemes: A general result. Econometrica 41, 4 (1973), 587-601.

A. Gibbard. 1977. Manipulation of schemes that mix voting with chance. Econometrica 45, 3 (1977), 665-681.

N. Golowich, H. Narasimhan, and D. C. Parkes. 2018. Deep Learning for Multi-Facility Location Mechanism Design. In Proceedings of the 27th International foint Conference on Artificial Intelligence (IFCAI). 261-267.

L. Mei, M. Li, D. Ye, and G. Zhang. 2016. Strategy-proof mechanism design for facility location games: Revisited. In Proceedings of the 15th International foint Conference on Autonomous Agents and Multi-Agent Systems (AAMAS).

H. Moulin. 1980. On Strategy-proofness and single peakedness. Public Choice 45, 4 (1980), 437-455.

N. Nisan and A. Ronen. 2001. Algorithmic Mechanism Design. Games and Economic Behavior 35, 1-2 (2001), 166-196.

M. Pál, É. Tardos, and T. Wexler. 2001. Facility location with nonuniform hard capacities. In Proceedings of the FOCS'01. 329-338.

W. Peremans, H. Peters, H. v.d. Stel, and T. Storcken. 1997. Strategy-proofness on Euclidean spaces. Social Choice and Welfare 14 (1997), 379-401.

A. D. Procaccia and M. Tennenholtz. 2013. Approximate Mechanism Design Without Money. In Proceedings of the 14th ACM Conference on Electronic Commerce (ACM-EC). ACM Press, 1-26.

M.A. Satterthwaite. 1975. Strategy-proofness and Arrow's conditions: Existence and correspondence theorems for voting procedures and social welfare functions. Fournal of Economic Theory 10 (1975), 187-217.

X. Sui and C. Boutilier. 2015. Approximately Strategy-proof mechanisms for (constrained) facility location. In Proceedings of the 14th International foint Conference on Autonomous Agents and Multi-Agent Systems (AAMAS). 605-613. 
J. Vygen. 2004. Approximation algorithms facility location problems. Lecture notes, Research Institute for Discrete Mathematics, University of Bonn, Germany.

J.A. Weymark. 2011. A unified approach to strategy-proofness for single-peaked preferences. SERIEs 2, 4 (2011), 529-550.

\section{A OMITTED PROOFS}

Proof of Proposition 3.1. Let $\langle\boldsymbol{x}, s, k\rangle$ be an arbitrary instance, and consider the subgame $\Gamma_{\boldsymbol{x}}(s, k)$. We first show there always exists an equilibrium where agents in $N_{k}^{*}(\boldsymbol{x}, s)$ are served, and the others are not. To see this, suppose all $i \in N_{k}^{*}(\boldsymbol{x}, s)$ choose action $a_{i}=s$ and all other agents choose $a_{i}=\emptyset$. In this case, the $k$ agents in $N_{k}^{*}(\boldsymbol{x}, s)$ attain utility $1-d\left(s, x_{i}\right) \geq 0$, and all other agents attain utility zero. An agent in $i \in N_{k}^{*}(\boldsymbol{x}, s)$ can never strictly benefit from deviating to $a_{i}=0$, since this leads to utility zero. An agent $j$ not in $N_{k}^{*}(x, s)$ can never strictly benefit from deviating to $a_{j}=s$, since the tie-breaking rule $(\triangleright)$ would lead to the agent not being served - hence, attaining utility $-d\left(s, x_{j}\right) \leq 0$.

We now show that an agent's equilibrium utility is invariant across equilibria (when multiple equilibria exist). Let $i \in N$ be some agent, let $\sigma, \sigma^{\prime}$ be two distinct equilibria of the subgame $\Gamma_{\boldsymbol{x}}(s, k)$. Denote agent $i$ 's utility in each of these equilibria by $\bar{u}_{i}, \bar{u}_{i}^{\prime}$, respectively. Note that $\bar{u}_{i}, \bar{u}_{i}^{\prime} \in$ $\left\{0,1-d\left(s, x_{i}\right)\right\}$.

For the sake of a contradiction suppose that $\bar{u}_{i} \neq \bar{u}_{i}^{\prime}$, notice that this necessarily implies that $d\left(s, x_{i}\right)<1$ and $k<n$. Without loss of generality assume $\bar{u}_{i}=0$ and $\bar{u}_{i}^{\prime}=1-d\left(s, x_{i}\right)>0$. If $\sigma$ is an equilibrium it must be that agent $i$ is not served when choosing action $a_{i}=s$; that is, agent $i$ is not in the set of $k$-closest agents $N_{k}^{*}(\boldsymbol{x}, s)$. Now consider the equilibrium $\sigma^{\prime}$ where agent $i$ is served. Given that the facility has capacity $k<n$, and agent $i$ is served despite $i \notin N_{k}^{*}(\boldsymbol{x}, s)$ there must be an agent $j \in N_{k}^{*}(\boldsymbol{x}, s)$ such that they choose action $a_{j}^{\prime}=\emptyset$ (and are not served). In this case, agent $j$ attain utility zero in equilibrium $\sigma^{\prime}$. If instead agent $j$ unilaterally deviated to the action $a_{j}^{\prime \prime}=s$ they would be served and attain utility $1-d\left(s, x_{j}\right) \geq 0$. Furthermore, $1-d\left(s, x_{j}\right)>0$ since agent $j \in N_{k}^{*}(\boldsymbol{x}, s)$ and $i \notin N_{k}^{*}(\boldsymbol{x}, s)$ and so $d\left(s, x_{j}\right) \leq d\left(s, x_{i}\right)<1$. We conclude that $\sigma^{\prime}$ is not an equilibrium; that is, we have a contradiction.

Proof of Proposition 3.2. Let $\langle\boldsymbol{x}, s, k\rangle$ and $\left\langle\boldsymbol{x}, \boldsymbol{s}^{\prime}, k\right\rangle$ be two instances such that for some agent $i s<s^{\prime} \leq x_{i}$, or $x_{i} \leq s^{\prime}<s$. From Proposition 3.1, in the first instance we know that agent $i$ attains utility $1-d\left(s, x_{i}\right)$ whenever $i \in N_{k}^{*}(\boldsymbol{x}, s)$ and otherwise attains utility zero. Similarly, in the second instance $i$ attains utility $1-d\left(s^{\prime}, x_{i}\right)$ whenever $i \in N_{k}^{*}\left(\boldsymbol{x}, s^{\prime}\right)$ and otherwise attains utility zero The set $N_{k}^{*}(\boldsymbol{x}, s)$ is defined as the $k$-closest agents with respect to $\triangleright$ to the facility, and $N_{k}^{*}\left(\boldsymbol{x}, s^{\prime}\right)$ is similarly defined with respect to the priority $\nabla^{\prime}$. If $s<s^{\prime} \leq x_{i}$ or $x_{i} \leq s^{\prime}<s$ then agent $i$ 's priority under $\triangleright^{\prime}$ (weakly) improves compared to their priority under $\triangleright$. Thus, $i \in N_{k}^{*}(\boldsymbol{x}, s)$ implies $i \in N_{k}^{*}\left(\boldsymbol{x}, s^{\prime}\right)$. We conclude that agent $i$ attains weakly higher utility in the $\left\langle\boldsymbol{x}, s^{\prime}, k\right\rangle$ instance. 